\title{
IDETC2020-22387
}

\section{DRAFT: BIFURCATION ANALYSIS OF A LANE KEEPING CONTROLLER WITH FEEDBACK DELAY}

\author{
Illés Vörös \\ Department of Applied Mechanics \\ Budapest University of Technology \\ and Economics \\ Budapest, $\mathrm{H}-1111$ \\ Hungary \\ Email: illes.voros@mm.bme.hu
}

\author{
Dénes Takács \\ MTA-BME Research Group on \\ Dynamics of Machines and Vehicles \\ Budapest, $\mathrm{H}-1111$ \\ Hungary \\ takacs@mm.bme.hu
}

\begin{abstract}
The aim of this study is to highlight nonlinear behaviors and periodic orbits of the single-track vehicle model with a delayed feedback controller. Two widely used tire models, namely a linear tire characteristic and Pacejka's Magic Formula are considered. Linearly stable domains of parameters such as the vehicle speed and the control gains are determined. Periodic solutions originating from Hopf bifurcation points are followed using numerical continuation and the results obtained with the two different tire models are compared. It is shown that neglecting the saturation of the tire lateral forces at total sliding might change the sense of certain Hopf bifurcations from subcritical to supercritical. The results are verified by numerical simulations. The resulting bifurcation diagrams aim to quantify the degree of robustness of these controllers with regards to the initial conditions at various parameter ranges in order to assure stable and safe operation.
\end{abstract}

\section{INTRODUCTION}

The lateral position control of the vehicle is a cornerstone of any advanced driver assistance system which involves the tracking of a trajectory. Lane keeping and lane changing functions, as well as the automatic execution of emergency maneuvers all require that the vehicle can reliably steer itself to follow a specific trajectory.

Widely used solutions to this problem include simple preview control [1], the pure pursuit [2] or the Stanley controller [3]. With advances in computational power and optimization algorithms, the real-time use of model predictive control is also becoming a viable option [4]. Nonlinear control techniques have also been successfully applied to control the lateral dynamics of the vehicle, such as feedback linearization [5] and differential flatness [6]. In this paper, a simple feedback controller is investigated with the explicit consideration of time delay. Sources of time delay in the closed-loop system may include sensor and communication delays [7], data processing time for lane detection and position estimation [8-10], as well as the non-modeled dynamics of the steering system $[3,11]$. Overall, when added up, the individual sources of delays can amount to several hundreds of milliseconds.

A crucial step to reliable and safe control design is understanding how the system reacts to large disturbances. This can be achieved with the methods of nonlinear analysis and bifurcation theory $[12,13]$. Because of its importance regarding road safety, the nonlinear behavior of vehicles has already been extensively studied in the literature [14-20]. In this paper, the bifurcation analysis of straight-line motion of the closed-loop system is performed with the help of numerical continuation [12,13,21], by considering the vehicle speed and the control gains as the bifur- 
cation parameter. Following the branches of periodic solutions originating from the linear stability limit paints a picture of what kinds of motions to expect due to large enough disturbances in different parameter ranges. In addition, we also consider a simpler, linear tire characteristic (while keeping the geometrical nonlinearities of the vehicle model), which is also widely used in control applications. Our aim is to understand how the choice of tire model affects the global dynamics of the system. Being aware of these differences is crucial for the design of robust model-based control solutions.

The rest of the paper is organized as follows: first, the equations of motion of the single-track vehicle model are presented. Two different options for tire modeling are considered, and the vehicle model is extended with a delayed feedback controller that generates the steering angle. Afterwards, the linear stability analysis of the closed-loop system is performed. The final section is devoted to the nonlinear analysis of the system, uncovering stable and unstable periodic solutions by varying the vehicle speed and the control gains. The results are verified by numerical simulations.

\section{MECHANICAL MODEL}

The well-known single-track vehicle model is considered in this study, with two different tire characteristics. In the final part of this section, the model is extended with lane-keeping control based on delayed state feedback.

\section{Single-Track Vehicle Model}

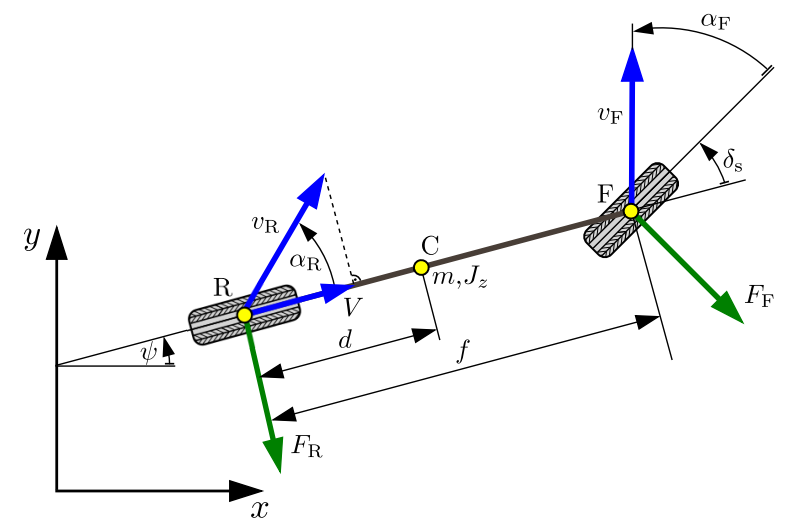

FIGURE 1. The single-track vehicle model (for further details, see $[22,23])$.

The main simplification of the single-track vehicle model (shown in Fig. 1) is that the tire contact patches are summarized along each axle and the vehicle body is modeled with respect to its longitudinal axis. Furthermore, the center of gravity is assumed to be on the ground level, therefore roll and pitch dynamics are neglected, and a constant longitudinal velocity $V$ is assumed. The governing equations are

$$
\begin{gathered}
\dot{x}=V \cos \psi-\sigma_{1} \sin \psi \\
\dot{y}=V \sin \psi+\sigma_{1} \cos \psi \\
\dot{\psi}=\sigma_{2} \\
m\left(\dot{\sigma}_{1}-V \sigma_{2}\right)=-\frac{d^{2} m+J_{z}}{J_{z}}\left(\sin \psi\left(F_{\mathrm{F}, x}+F_{\mathrm{R}, x}\right)\right. \\
\left.-\cos \psi\left(F_{\mathrm{F}, y}+F_{\mathrm{R}, y}\right)\right)+\frac{f d m}{J_{z}}\left(\sin \psi F_{\mathrm{F}, x}-\cos \psi F_{\mathrm{F}, y}\right) \\
J_{z} \dot{\sigma}_{2}=\sin \psi\left((d-f) F_{\mathrm{F}, x}+d F_{\mathrm{R}, x}\right)-\cos \psi\left((d-f) F_{\mathrm{F}, y}+d F_{\mathrm{R}, y}\right)
\end{gathered}
$$

where the state variables are the global coordinates $x$ and $y$ of the rear axle (point $\mathrm{R}$ ), the vehicle heading $\psi$, the lateral velocity $\sigma_{1}$ of point $\mathrm{R}$, and the yaw rate $\sigma_{2}$. The vehicle parameters include the mass $m$, the yaw moment of inertia $J_{z}$ around the center of gravity $\mathrm{C}$, the wheelbase $f$, and the distance $d$ between the rear axle and the center of gravity. The tire side forces are denoted by $F_{i, j}$, where the first index represents the front or the rear axle ( $\mathrm{F}$ or $\mathrm{R}$, respectively), and the second index denotes the $x$ or $y$ force component in the global coordinate system. For the detailed derivation of the above equations using the Gibbs-Appellmethod [24, 25], the reader is referred to [26].

\section{Tire Models}

Since our focus is on the lateral dynamics of the vehicle in case of constant longitudinal speed, longitudinal tire forces are not considered here. Moreover, the self-aligning moments generated by the tire side forces are also neglected, due to the insignificant moment arms compared to the vehicle geometry. The side forces are modeled as a function of the side slip angles $\alpha_{\mathrm{F}}$ and $\alpha_{\mathrm{R}}$ :

$$
\begin{aligned}
\alpha_{\mathrm{F}} & =\arctan \left(\frac{\dot{y}+f \cos \psi \dot{\psi}}{\dot{x}-f \sin \psi \dot{\psi}}\right)-\psi-\delta_{\mathrm{s}}, \\
\alpha_{\mathrm{R}} & =\arctan \left(\frac{\dot{y}}{\dot{x}}\right)-\psi
\end{aligned}
$$

where $\delta_{\mathrm{s}}$ denotes the steering angle. A common simplification is often used for the tire forces, namely, a linear relationship between the side slip angles and the side forces can be considered, leading to

$$
F_{i}^{\mathrm{lat}}=\tilde{C}_{i} \alpha_{i}, \quad i \in\{\mathrm{F}, \mathrm{R}\}
$$


where $\tilde{C}_{i}$ is the so-called cornering stiffness. The superscript lat refers to the fact that the above represents the overall side forces acting on the wheels, prior to decomposing them into their $x$ and $y$ components, as in Eqn. (4) and (5).

The above model is a good approximation for small side-slip angles, but it fails to capture the saturation of the tire forces when total sliding starts. Therefore the other tire model considered in this study is Pacejka's Magic Formula [22], which calculates the tire side forces as

$$
F_{i}^{\text {lat }}=D_{i} \sin \left(C_{i} \arctan \left(B_{i} \alpha_{i}-E_{i}\left(B_{i} \alpha_{i}-\arctan \left(B_{i} \alpha_{i}\right)\right)\right)\right) .
$$

This model is also widely used for both simulation and control purposes, since it is capable of reproducing the distinct shape of measured tire characteristics in a single continuous function. The parameters $B_{i}, C_{i}, D_{i}$ and $E_{i}$ are usually determined by curve fitting. Linearizing the Magic Formula around zero side slip angle leads to the relationship $\tilde{C}_{i}=B_{i} C_{i} D_{i}$ between the parameters of the two models.

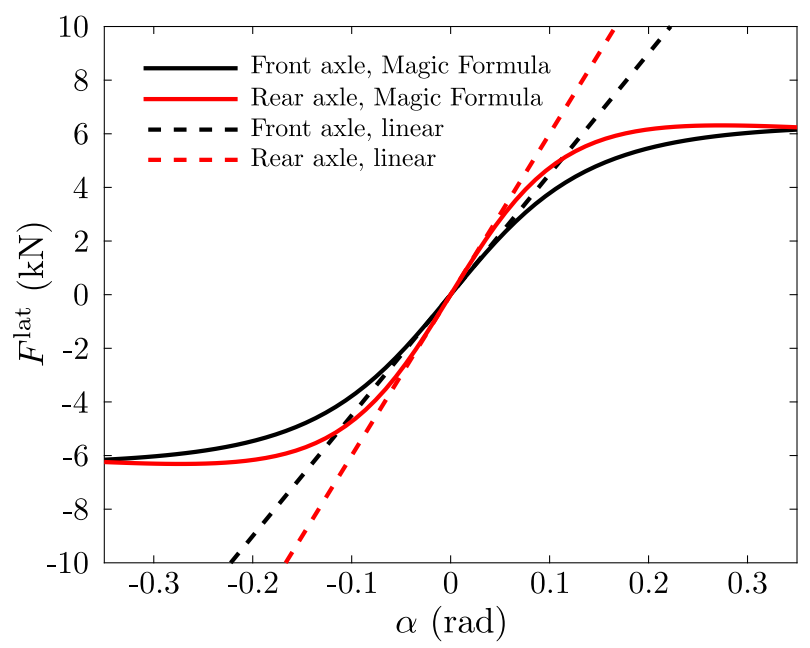

FIGURE 2. Tire characteristics using the Magic Formula with parameters listed in Table 1 (continuous), and its linear approximation (dashed). Black: front axle, red: rear axle.

In this paper, an understeering vehicle setup is considered, with parameter values of the Magic Formula listed in Table 1 (the rest of the vehicle parameters are listed in Table 2). The parameters $D_{\mathrm{F}}$ and $D_{\mathrm{R}}$ are determined by the corresponding vertical wheel loads, which were calculated assuming a friction coefficient of 0.9 at both axles. The cornering stiffness values of the corresponding linear model are $\tilde{C}_{\mathrm{F}}=B_{\mathrm{F}} C_{\mathrm{F}} D_{\mathrm{F}}=45 \mathrm{kN}$ and $\tilde{C}_{\mathrm{R}}=B_{\mathrm{R}} C_{\mathrm{R}} D_{\mathrm{R}}=60 \mathrm{kN}$. The tire characteristics are plotted in Fig. 2.
TABLE 1. Parameter Values of the Magic Formula Tire Model for the Front (F) and Rear (R) Axle

\begin{tabular}{cccccccc}
\hline$B_{\mathrm{F}}$ & $B_{\mathrm{R}}$ & $C_{\mathrm{F}}$ & $C_{\mathrm{R}}$ & $D_{\mathrm{F}}$ & $D_{\mathrm{R}}$ & $E_{\mathrm{F}}$ & $E_{\mathrm{R}}$ \\
\hline 5.940 & 6.336 & 1.2 & 1.5 & $6313 \mathrm{~N}$ & $6313 \mathrm{~N}$ & 0 & 0 \\
\hline
\end{tabular}

TABLE 2. Vehicle Parameter Values

\begin{tabular}{lll}
\hline Parameter & Notation & Value \\
\hline Vehicle wheelbase & $f$ & $2.7 \mathrm{~m}$ \\
$\begin{array}{l}\text { Distance between rear } \\
\text { axle and center of gravity }\end{array}$ & $d$ & $1.35 \mathrm{~m}$ \\
Vehicle mass & $m$ & $1430 \mathrm{~kg}$ \\
Yaw moment of inertia & $J_{z}$ & $2500 \mathrm{kgm}^{2}$ \\
\hline
\end{tabular}

\section{Lane-Keeping Control}

The goal of the lane-keeping controller is to guide the vehicle to the center of the lane. To this end, the steering angle is generated proportionally to the lateral position error and the orientation error with respect to the lane centerline. Without loss of generality, the reference path in this paper is going to be a straight line at $y=0$, leading to the control law

$$
\delta_{\mathrm{s}}(t)=-P_{y} y(t-\tau)-P_{\psi} \psi(t-\tau)
$$

where $P_{y}$ and $P_{\psi}$ are the control gains, and the time delay $\tau$ includes sensor and communication delays, processing time as well as the dynamics of the actuators and the steering mechanism.

\section{LINEAR STABILITY ANALYSIS}

In case of the uncontrolled system, the coordinates $x, y$ and the yaw angle $\psi$ can be decoupled from the rest of the state variables, since the dynamics of the vehicle are independent from its position and orientation in the plane. However, once position control is introduced, this is not true anymore: due to feedback control, the dynamics will also depend on the vehicle's position relative to the desired path. Since in this analysis we only consider motion along the $x$-axis, the position error can be calculated based on the $y$ coordinate alone. Control law (10) also requires feedback of the orientation error, but the state variable $x$ can still be decoupled from the rest and further calculations can be based on Eqn. (2)-(5).

The equilibrium of straight ahead motion along the $x$ axis corresponds to the state vector $\mathbf{q}=\left[\begin{array}{llll}y & \psi & \sigma_{1} & \sigma_{2}\end{array}\right]^{\mathrm{T}}=\mathbf{0}$. Linearizing the system around this equilibrium leads to the following state space representation:

$$
\dot{\mathbf{q}}(t)=\mathbf{A q}(t)+\mathbf{B u}(t-\tau),
$$



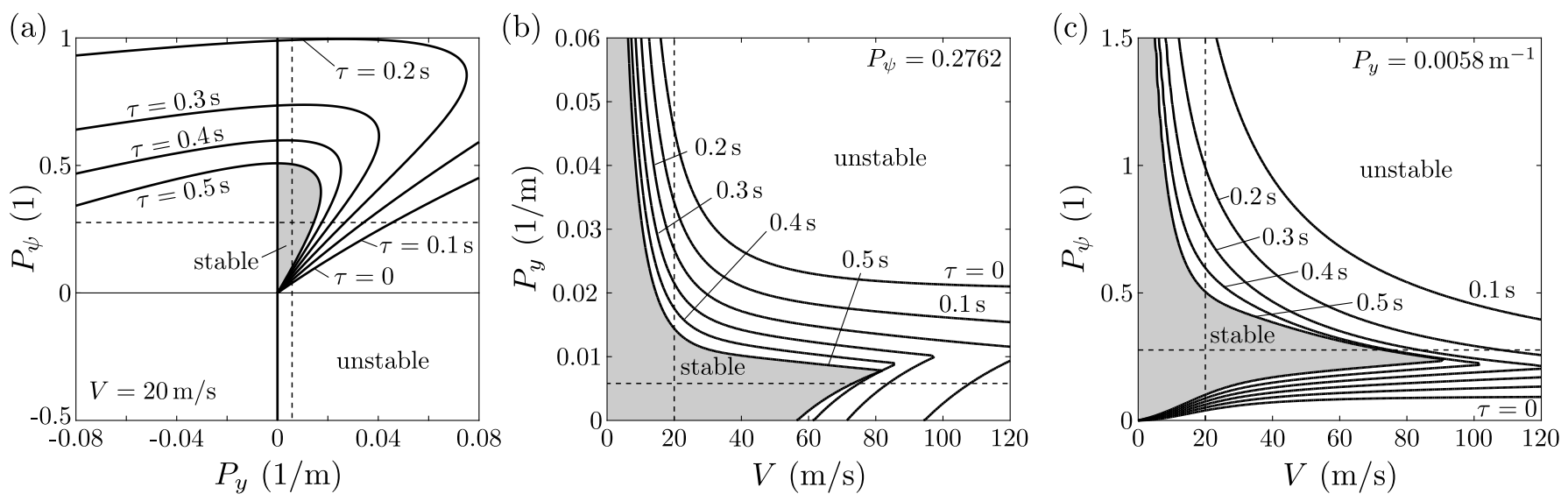

FIGURE 3. Linearly stable parameter regions of straight-line motion for various amounts of time delay.

with the system and input matrices

$$
\mathbf{A}=\left[\begin{array}{cccc}
0 & V & 1 & 0 \\
0 & 0 & 0 & 1 \\
0 & 0 & A_{33} & A_{34} \\
0 & 0 & A_{43} & A_{44}
\end{array}\right], \quad \mathbf{B}=\left[\begin{array}{c}
0 \\
0 \\
B_{3} \\
B_{4}
\end{array}\right],
$$

with elements

$$
\begin{gathered}
A_{33}=-\frac{B_{3}}{V}-\frac{\tilde{C}_{\mathrm{R}}\left(d^{2} m+J_{z}\right)}{m V J_{z}}, \quad A_{34}=-B_{3} \frac{f}{V}-V, \\
A_{43}=-\frac{B_{4}}{V}+\frac{\tilde{C}_{\mathrm{R}} d}{V J_{z}}, \quad A_{44}=-B_{4} \frac{f}{V},
\end{gathered}
$$

and

$$
B_{3}=\frac{\tilde{C}_{\mathrm{F}}\left(d(d-f) m+J_{z}\right)}{m J_{z}}, \quad B_{4}=\frac{\tilde{C}_{\mathrm{F}}(f-d)}{J_{z}} .
$$

Using the delayed state feedback controller in Eqn. (10), the system input can be written as $\mathbf{u}(t-\tau)=\mathbf{K q}(t-\tau)$ with the gain vector $\mathbf{K}=\left[\begin{array}{lll}-P_{y}-P_{\psi} & 0 & 0\end{array}\right]$. The characteristic equation of the closed-loop linear system can then be written as

$$
D(\lambda):=\operatorname{det}\left(\lambda \mathbf{I}-\mathbf{A}-\mathbf{B K} \mathrm{e}^{-\lambda \tau}\right)=0,
$$

where $\lambda \in \mathbb{C}$ denotes the characteristic exponent and $\mathbf{I}$ is the identity matrix. The exact form of $D(\lambda)$ can be found in [26].

The linear stability of the system can be checked using e.g. the Routh-Hurwitz-criterion [24] for the delay-free case, and with the help of the D-subdivision or the semi-discretizetion method in the delayed case [27]. The resulting stability maps can be seen in Fig. 3. These show the stable parameter regions in terms of the control gains $P_{y}$ and $P_{\psi}$, as well as the vehicle speed $V$, for various amounts of time delay. Figure 3(a) corresponds to $V=20 \mathrm{~m} / \mathrm{s}$. In Fig. 3(b), $P_{\psi}$ is fixed at 0.2762 , while Fig. 3(c) was plotted for $P_{y}=0.00581 / \mathrm{m}$. These control gains provide the most highly damped system response at the speed of $20 \mathrm{~m} / \mathrm{s}$, assuming a time delay of $\tau=0.4 \mathrm{~s}$. The bifurcation diagrams in the next section are plotted along these values, denoted by dashed lines in Fig. 3.

\section{BIFURCATION ANALYSIS}

The stability maps in Fig. 3 only give us information about the local stability of straight-line motion. It is possible, however, that depending on the initial conditions (or as a result of large enough disturbances), the system exhibits a qualitatively different behavior than what the stability maps may suggest. In order to uncover what kinds of system dynamics are possible near the linearly stable regions, the methods of bifurcation theory are applied $[12,13,28]$. In particular, we use the numerical continuation capabilities of the Matlab package DDE-Biftool $[21,29,30]$ to follow branches of periodic solutions emerging from Hopf bifurcation points. The periodic orbits in DDE-Biftool are calculated using orthogonal collocation with piecewise polynomials. This is particularly important for unstable periodic solutions, since direct numerical methods cannot be used in such cases. For further details, the reader is referred to [31] and the references in [30].

In order to help visualize the corresponding dynamics, the amplitude of the lateral displacement of the arising periodic motions will be shown as a function of the bifurcation parameter (either the vehicle speed, or one of the control gains). However, the domains of attraction of the stable solutions depend on the rest of the variables too (note the infinite dimensional phase space of the delayed system), therefore the resulting bifurcation diagrams 
are not sufficient to determine the critical lateral displacement of the vehicle above which it loses its stability. Nevertheless, they still provide guidelines about which parameter regions might be more dangerous than the rest.

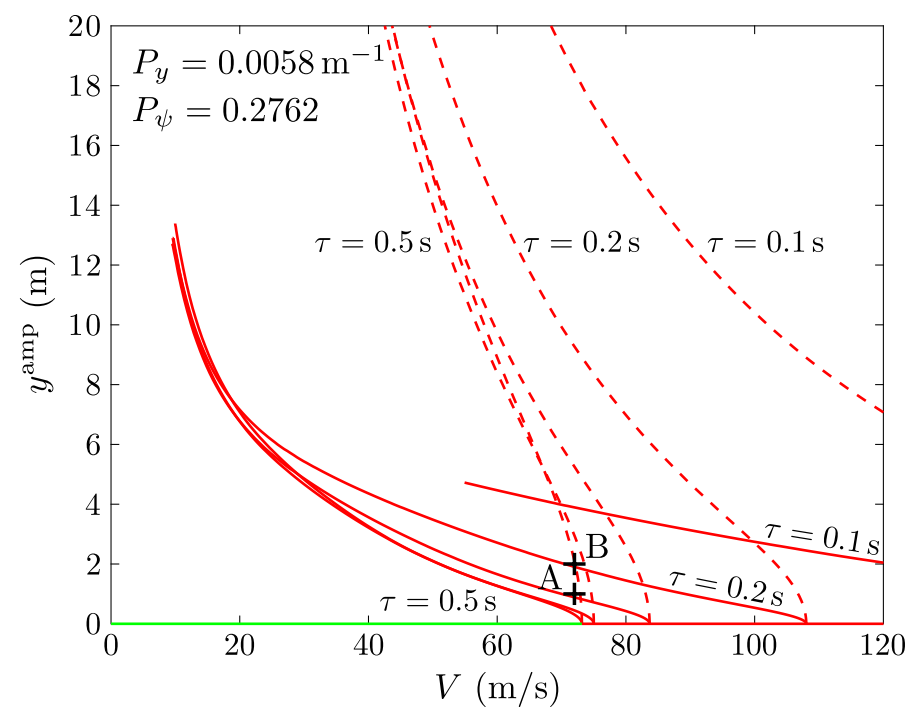

FIGURE 4. Bifurcation diagram showing the amplitude of the lateral displacement of the vehicle as a function of speed, for various amounts of time delay $\left(P_{y}=0.0058 \mathrm{1} / \mathrm{m}, P_{\psi}=0.2762\right)$. Continuous lines are calculated using the Magic Formula and dashed lines refer to the linear tire model. The coloring of the $y^{\mathrm{amp}}=0$ solution shows the stability of the equilibrium for $\tau=0.5 \mathrm{~s}$ (green: stable, red: unstable).

First, the vehicle speed $V$ is used as the bifurcation parameter, while fixing the control gains at $P_{y}=0.00581 / \mathrm{m}$ and $P_{\psi}=0.2762$ (see the horizontal dashed lines in panels (b) and (c) of Fig. 3). The resulting bifurcation diagram can be seen in Fig. 4. It shows that at the point of stability loss, a subcritical Hopf bifurcation occurs, leading to an unstable periodic orbit around the locally stable equilibrium. This means that certain initial conditions can lead to unstable vehicle motion even below the critical speed. However, the criticality of the arising Hopf bifurcation also depends on other parameters, such as the vehicle and tire configuration as well as the control gains. As a result, both a subcritical or a supercritical (as in e.g. [16]) Hopf bifurcation may be observed when increasing the speed of an understeering vehicle in case of delayed feedback control. Uncovering the exact dependence of the periodic solutions on these aspects is the subject of further research.

According to Fig. 4, there is a major difference between the two tire models regarding the amplitude of the periodic solutions at certain vehicle speeds. This difference is illustrated in the numerical simulations of Fig. 5. Choosing a linearly stable vehicle speed $(V=72 \mathrm{~m} / \mathrm{s})$ close to the Hopf bifurcation point at
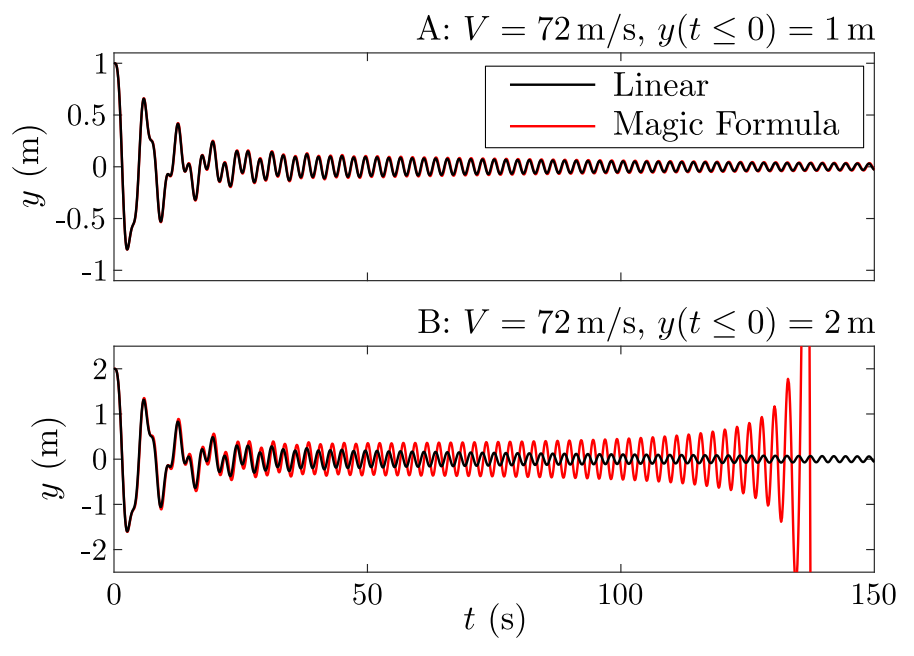

FIGURE 5. Simulation results near the critical speed of $V=73.2 \mathrm{~m} / \mathrm{s}$ where Hopf-bifurcation occurs for $\tau=0.5 \mathrm{~s}$ (see points A and B in Fig. 4). Because of the smaller amplitude unstable limit cycle, the nonlinear tire model is more sensitive to the initial conditions.

$V=73.2 \mathrm{~m} / \mathrm{s}$ (for $\tau=0.5 \mathrm{~s}$ ), the simulations were run from two different initial conditions, shown as points $\mathrm{A}$ and $\mathrm{B}$ in Fig. 4. First, starting from a lateral displacement of $y(t \leq 0)=1 \mathrm{~m}$ (while the other state variables are 0 ), both tire models lead to stable straight ahead motion. However, when the initial displacement is increased to $y(t \leq 0)=2 \mathrm{~m}$, the vehicle with the Magic Formula starts oscillating with increasing amplitude, even though the longitudinal velocity is below the critical value. Although the above speed values are rather high with limited practical relevance, but the point of these simulations is to verify the differences between the two tire models.

In the bifurcation diagram of Fig. 6, the control gain $P_{y}$ is used as the bifurcation parameter, while $P_{\psi}$ is fixed at 0.2762 and $V=20 \mathrm{~m} / \mathrm{s}$ (see the horizontal dashed line in Fig. 3(a), or the vertical line in Fig. 3(b)). With increasing time delay, the upper limit of $P_{y}$ decreases. In this case (for lower values of $\tau$ ), the criticality of the Hopf bifurcation depends on the choice of tire model: with a linear tire characteristic, stable periodic solutions arise from the stability boundaries, while the Magic Formula leads to a subcritical Hopf bifurcation. Therefore, the linearly stable parameter domain might appear to be globally stable when using a simpler tire model, while in fact the saturation of the tire forces limit the domain of attraction of the stable equilibrium. For $\tau=0.4 \mathrm{~s}$, the periodic branch of the Magic Formula starts off as supercritical, but it shortly changes direction through a fold bifurcation point, forming an unstable limit cycle around the stable equilibrium.

The corresponding numerical simulations can be seen in Fig. 7: when the simulations are started from a smaller initial displacement of $y(0)=0.5 \mathrm{~m}$ with a linearly stable control gain $\left(P_{y}=0.0451 / \mathrm{m}\right.$; denoted as point $\mathrm{C}$ in Fig. 6$)$, the vehicle is sta- 


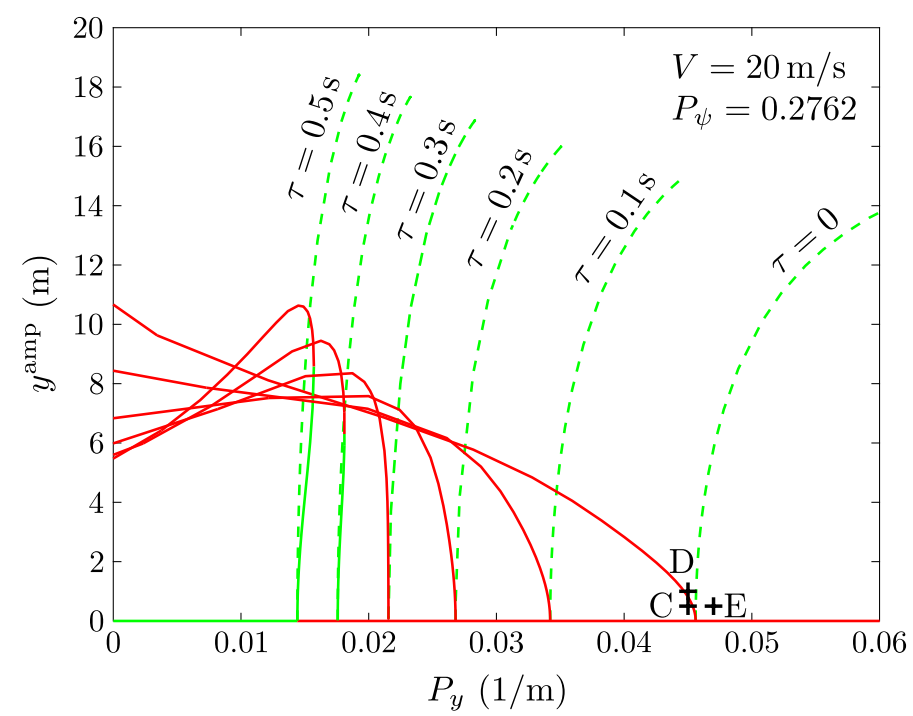

FIGURE 6. Bifurcation diagram showing the amplitude of the lateral displacement of the vehicle as a function of the control gain $P_{y}$, for various amounts of time delay $\left(P_{\psi}=0.2762, V=20 \mathrm{~m} / \mathrm{s}\right)$. Continuous: Magic Formula, dashed: linear tire model. The coloring of the $y^{\mathrm{amp}}=0$ solution shows the stability of the equilibrium for $\tau=0.5 \mathrm{~s}$ (green: stable, red: unstable).
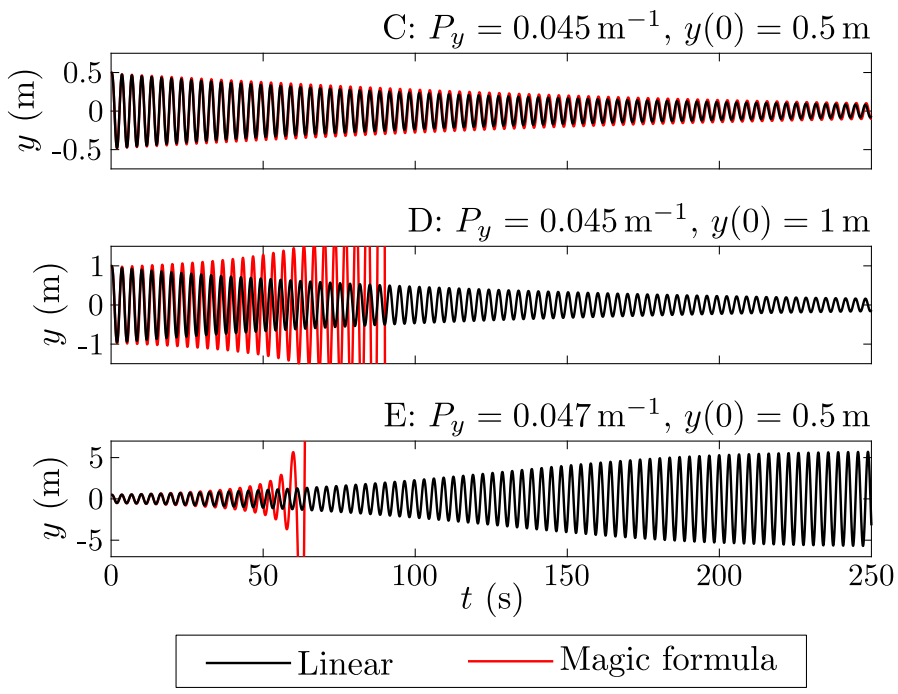

FIGURE 7. Simulation results near the stability boundary of $P_{y}=$ $0.04561 / \mathrm{m}$ where Hopf-bifurcation occurs for $\tau=0$ (see points C, D and $\mathrm{E}$ in Fig. 6). Choosing the control gain outside the linearly stable region leads to a globally unstable solution with the Magic Formula, while the vehicle with linear tire characteristics finds a stable periodic orbit.

ble with both tire models. However, when the initial condition is increased to $y(0)=1 \mathrm{~m}$ (point $\mathrm{D}$ ), the vehicle with the Magic
Formula produces violent oscillations due to the presence of the unstable limit cycle. If the control gain is chosen from the linearly unstable region $\left(P_{y}=0.0471 / \mathrm{m}\right.$; point $\left.\mathrm{E}\right)$, then the solution using the linear tire model tends to a stable periodic orbit, while no periodic solution is found using the Magic Formula.
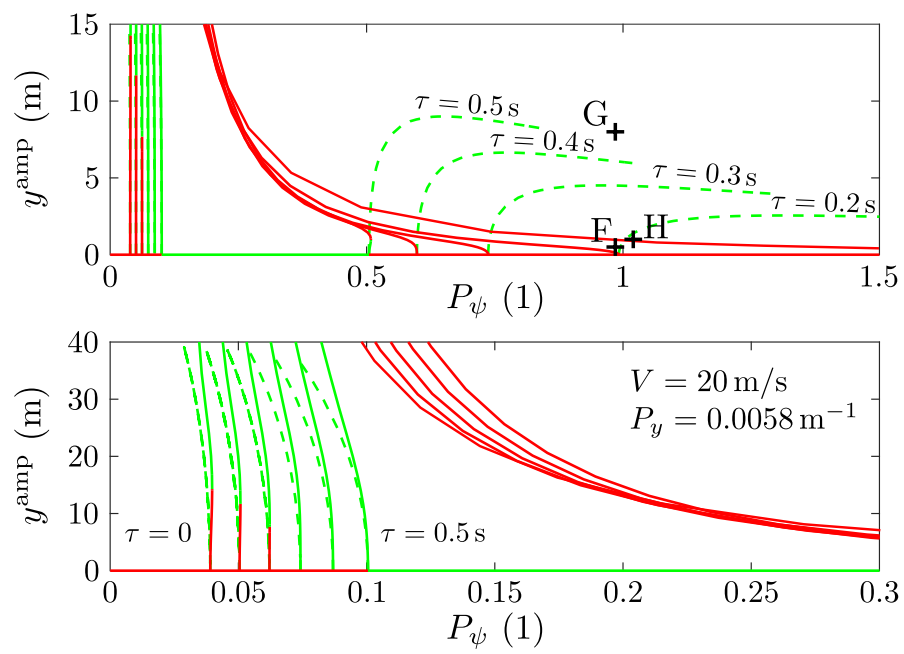

FIGURE 8. Bifurcation diagram showing the amplitude of the lateral displacement of the vehicle as a function of the control gain $P_{\psi}$, for various amounts of time delay $\left(P_{y}=0.00581 / \mathrm{m}, V=20 \mathrm{~m} / \mathrm{s}\right)$. Continuous: Magic Formula, dashed: linear tire model. The coloring of the $y^{\mathrm{amp}}=0$ solution shows the stability of the equilibrium for $\tau=0.5 \mathrm{~s}$ (green: stable, red: unstable).

The bifurcation diagram in Fig. 8 shows that increasing $P_{\psi}$ around the (upper) edge of stability has a similar effect to increasing $P_{y}$ : when using a linear tire model, a supercritical Hopf bifurcation can be observed, while the Magic Formula leads to a subcritical bifurcation (which can also start off as supercritical for large enough delays). However, unlike $P_{y}$, choosing a too low value for the gain $P_{\psi}$ can also lead to stability loss (see Fig. 3 for a different perspective). Here, another Hopf bifurcation occurs, which is generally supercritical for both tire models, but in the case of the Magic Formula, it starts off as slightly subcritical for lower delay values.

Based on Fig. 8, the designer should be particularly careful when choosing the gain of the orientation error. As an example, the linear stability limit for the case of $\tau=0.2 \mathrm{~s}$ is at $P_{\psi}=0.99$, but the amplitude of the unstable limit cycle is well within the width of a single lane even at $P_{\psi}=0.6$. Although this is a single example only, it still shows that depending on the parameters, there can be dangerous ranges of the control gains far within the linearly stable domain that should be avoided.

Figure 9 shows the verification of the stable and unstable limit cycles in Fig. 8 with numerical simulations (for $\tau=0.2 \mathrm{~s}$ ). 

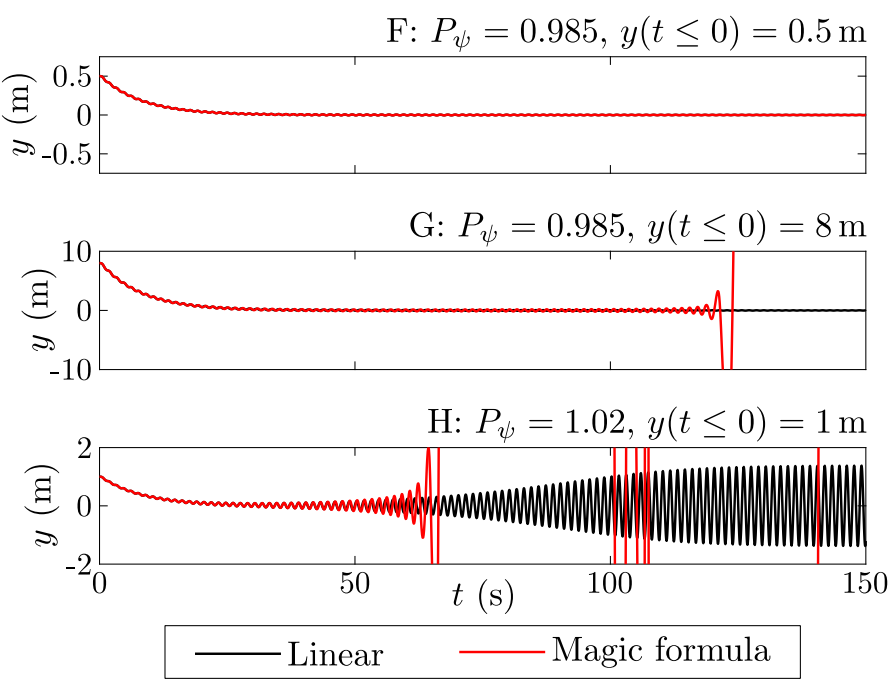

FIGURE 9. Simulation results near the stability boundary of $P_{\psi}=$ 0.99 where Hopf-bifurcation occurs for $\tau=0.2 \mathrm{~s}$ (see points $\mathrm{F}, \mathrm{G}$ and $\mathrm{H}$ in Fig. 8).

First, the gain was set to $P_{\psi}=0.985$. Although this is below the critical value of 0.99 , the vehicle with the Magic Formula can still lose its stability depending on the initial conditions. The simulation starting in point $\mathrm{H}$ shows the stable limit cycle of the linear tire model and the global instability of the Magic Formula at the linearly unstable value of $P_{\psi}=1.02$.

\section{CONCLUSION}

A lane-keeping controller with explicit consideration of feedback delay was analyzed in this paper. With the help of numerical continuation, periodic orbits around the stable straightline motion were highlighted. It was shown that neglecting the saturation of lateral tire forces leads to qualitatively different results, changing the criticality of certain Hopf bifurcations from subcritical to supercritical. It was also shown that (based on Fig. 4), it is very easy to largely overestimate the domain of attraction of the stable solution using only the linear tire model, which is important to keep in mind from a safety point of view.

Although the amplitude of most of the detected periodic motions are too large to be observed under normal road conditions, the bifurcation diagrams still provide useful guidelines about which parameter regions are more robust against disturbances. Moreover, it should be noted that our analysis was more on the conservative side in terms of two aspects: on the one hand, an understeering vehicle configuration was considered, which is inherently more stable than an oversteering one. On the other hand, the controller was tuned using a less aggressive tuning method. A more dynamic response can be reached by increasing the control gains, and other tuning strategies (e.g. optimizing some kind of cost function) could easily lead to larger gains. In such cases, the amplitude of the arising periodic solutions are expected to become lower (as in Fig. 8), making the consideration of nonlinear behavior even more important in order to ensure a stable and safe operation.

\section{ACKNOWLEDGMENT}

This research was partly supported by the National Research, Development and Innovation Office under grant no. NKFI-128422 and by the Higher Education Excellence Program of the Ministry of Human Capacities in the frame of Artificial intelligence research area of Budapest University of Technology and Economics (BME FIKP-MI).

\section{REFERENCES}

[1] Plöchl, M., and Edelmann, J., 2007. "Driver models in automobile dynamics application”. Vehicle System Dynamics, 45(7-8), pp. 699-741.

[2] Amidi, O., and Thorpe, C. E., 1991. "Integrated mobile robot control". In Mobile Robots V, Vol. 1388, International Society for Optics and Photonics, pp. 504-524.

[3] Hoffmann, G. M., Tomlin, C. J., Montemerlo, M., and Thrun, S., 2007. "Autonomous automobile trajectory tracking for off-road driving: Controller design, experimental validation and racing”. In American Control Conference, 2007. ACC'07, IEEE, pp. 2296-2301.

[4] Falcone, P., Eric Tseng, H., Borrelli, F., Asgari, J., and Hrovat, D., 2008. "Mpc-based yaw and lateral stabilisation via active front steering and braking". Vehicle System Dynamics, 46(S1), pp. 611-628.

[5] Liaw, D.-C., and Chung, W.-C., 2008. "A feedback linearization design for the control of vehicle's lateral dynamics". Nonlinear Dynamics, 52(4), pp. 313-329.

[6] Fuchshumer, S., Schlacher, K., and Rittenschober, T., 2005. "Nonlinear vehicle dynamics control-a flatness based approach". In Proceedings of the 44th IEEE Conference on Decision and Control, IEEE, pp. 6492-6497.

[7] Wang, R., Jing, H., Wang, J., Chadli, M., and Chen, N., 2016. "Robust output-feedback based vehicle lateral motion control considering network-induced delay and tire force saturation". Neurocomputing, 214, pp. 409-419.

[8] Wu, P.-C., Chang, C.-Y., and Lin, C. H., 2014. "Lane-mark extraction for automobiles under complex conditions". Pattern Recognition, 47(8), pp. 2756-2767.

[9] Abramov, A., Bayer, C., Heller, C., and Loy, C., 2016. "Multi-lane perception using feature fusion based on graphslam". In 2016 IEEE/RSJ International Conference on Intelligent Robots and Systems (IROS), IEEE, pp. 31083115 .

[10] Wang, Z., Ren, W., and Qiu, Q., 2018. "Lanenet: Real-time 
lane detection networks for autonomous driving". arXiv preprint arXiv:1807.01726.

[11] Mobus, R., and Zomotor, Z., 2005. "Constrained optimal control for lateral vehicle guidance". In IEEE Proceedings. Intelligent Vehicles Symposium, 2005., IEEE, pp. 429434.

[12] Kuznetsov, Y. A., 2013. Elements of applied bifurcation theory, Vol. 112. Springer Science \& Business Media.

[13] Strogatz, S. H., 2018. Nonlinear dynamics and chaos: with applications to physics, biology, chemistry, and engineering. CRC press.

[14] Ono, E., Hosoe, S., Tuan, H. D., and Doi, S., 1998. "Bifurcation in vehicle dynamics and robust front wheel steering control". IEEE transactions on control systems technology, 6(3), pp. 412-420.

[15] Shen, S., Wang, J., Shi, P., and Premier, G., 2007. "Nonlinear dynamics and stability analysis of vehicle plane motions". Vehicle System Dynamics, 45(1), pp. 15-35.

[16] Della Rossa, F., and Mastinu, G., 2018. "Analysis of the lateral dynamics of a vehicle and driver model running straight ahead". Nonlinear Dynamics, 92(1), pp. 97-106.

[17] Rossa, F. D., Mastinu, G., and Piccardi, C., 2012. "Bifurcation analysis of an automobile model negotiating a curve". Vehicle system dynamics, 50(10), pp. 1539-1562.

[18] Liu, Z., Payre, G., and Bourassa, P., 2004. "Stability and oscillations in a time-delayed vehicle system with driver control". Nonlinear Dynamics, 35(2), pp. 159-173.

[19] Mastinu, G., Biggio, D., Della Rossa, F., and Fainello, M., 2020. "Straight running stability of automobiles: experiments with a driving simulator". Nonlinear Dynamics, pp. 1-18.

[20] Várszegi, B., Takács, D., and Orosz, G., 2019. "On the nonlinear dynamics of automated vehicles-a nonholonomic approach". European Journal of Mechanics-A/Solids, 74, pp. 371-380.

[21] Engelborghs, K., Luzyanina, T., and Roose, D., 2002. "Numerical bifurcation analysis of delay differential equations using dde-biftool". ACM Transactions on Mathematical Software (TOMS), 28(1), pp. 1-21.

[22] Pacejka, H. B., 2006. Tyre and Vehicle Dynamics. Butterworth-Heinemann, Oxford.

[23] Rajamani, R., 2006. Vehicle Dynamics and Control. Springer.

[24] Gantmacher, F., 1975. Lectures in Analytical Mechanics. MIR Publiser, Moscow.

[25] Greenwood, D. T., 2006. Advanced Dynamics. Cambridge University Press.

[26] Vörös, I., Várszegi, B., and Takács, D., 2019. "Lane keeping control using finite spectrum assignment with modeling errors". Dynamic Systems and Control Conference, ASME. V003T18A002.

[27] Insperger, T., and Stépán, G., 2011. Semi-Discretization for
Time-Delay Systems. Springer.

[28] True, H., 2013. "Multiple attractors and critical parameters and how to find them numerically: the right, the wrong and the gambling way". Vehicle system dynamics, 51(3), pp. 443-459.

[29] Engelborghs, K., Luzyanina, T., and Samaey, G., 2001. "Dde-biftool v. 2.00: a matlab package for bifurcation analysis of delay differential equations". TW Reports.

[30] Sieber, J., Engelborghs, K., Luzyanina, T., Samaey, G., and Roose, D., 2014. "Dde-biftool manual-bifurcation analysis of delay differential equations". arXiv:1406.7144.

[31] Engelborghs, K., Luzyanina, T., Hout, K. I., and Roose, D., 2001. "Collocation methods for the computation of periodic solutions of delay differential equations". SIAM Journal on Scientific Computing, 22(5), pp. 1593-1609. 